\title{
Mitteilungen der DGIM
}

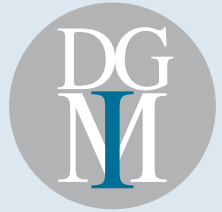

Deutschen Gesellschaft

für Innere Medizin e.V. (DGIM),

Irenenstraße 1,

D-65189 Wiesbaden,

Tel. 0611/205 8040-0 Fax 0611/205 8040-46

info@dgim.de

Diabetologe 2011 · 7:527-527

DOI 10.1007/s11428-011-0811-z

(c) Springer-Verlag 2011

Redaktion

U.R. Fölsch, Kiel

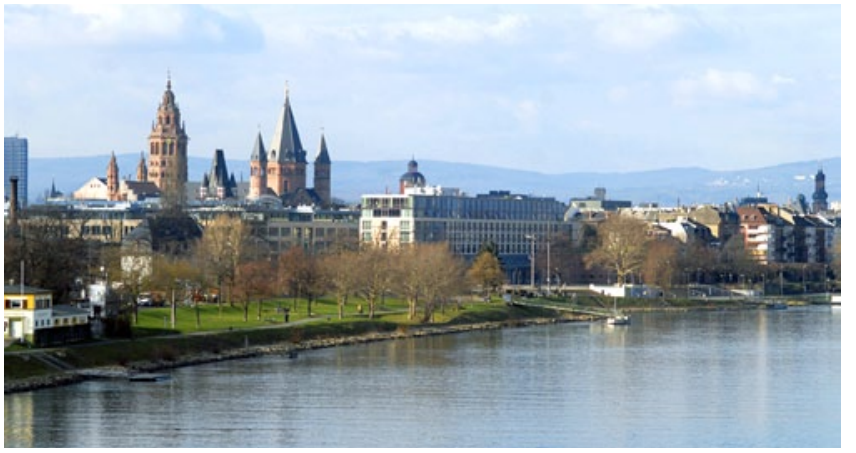

\section{Diabetes Update 2012 - jetzt interaktiv mit Diabetes eUpdate}

\author{
2. und 3. März 2012, Mainz
}

\section{Schirmherrschaft DGIM}

Am 2./3. März 2012 findet erneut in Mainz das bewährte Diabetes Update statt. Teilnehmern wird hier die Chance geboten, sich im Bereich der Diabetologie innerhalb von zwei Tagen auf den aktuellen Stand des Wissens zu bringen: Das Expertenteam präsentiert die wichtigsten aktuellen Studien des letzten Jahres und bewertet die Ergebnisse kritisch in Hinblick auf ihre praktische Relevanz. Teilnehmer haben während der Vorträge sowie im Anschluss die Möglichkeit, mit den Experten und Kollegen die neuen Sachverhalte zu diskutieren.

Jeder Teilnehmer des Diabetes Updates erhält das Handbuch DIABETOLOGIE 2012 mit Zusammenfassungen aller wichtigen Neu in diesem Jahr ist das Diabetes eUpdate: Auf dieser InternetPlattform kann auf alle Vorträge der Veranstaltung in Bild und Ton zugegriffen werden (für Teilnehmer kostenlos).

Das Team-Ticket steht nun auch Teilnehmern aus der Klinik zur Verfügung: Ärzte aus Klinik und Praxis können sich als Team (Arzt mit Assistenz) zu vergünstigten Konditionen anmelden - so wird das ganze Team auf den neuesten Stand gebracht.

Partner des Diabetes Updates ist seit Beginn der forschende Arzneimittelhersteller Boehringer Ingelheim, der das in Inhalt und Programmplanung unabhängige Konzept auch 2012 weiter unterstützt.
Publikationen des letzten Jahres.

\begin{tabular}{|c|c|c|}
\hline \multicolumn{3}{|c|}{ Programm } \\
\hline 09:45 & $\begin{array}{l}\text { Pathophysiologie und } \\
\text { Prävention }\end{array}$ & Prof. Stephan Martin, Düsseldorf \\
\hline $10: 30$ & $\begin{array}{l}\text { Diagnose, Epidemio- } \\
\text { logie, Screening }\end{array}$ & Dr. Wolfgang Rathmann, Düsseldorf \\
\hline $11: 45$ & $\begin{array}{l}\text { Typ } 1 \text { Diabetes + } \\
\text { Insulintherapie }\end{array}$ & Dr. Thorsten Siegmund, München \\
\hline $13: 00$ & Diabetes-Technologien & Prof. Lutz Heinemann, Neuss \\
\hline $14: 45$ & Adipositas & $\begin{array}{l}\text { Prof. Andreas Hamann, } \\
\text { Bad Nauheim }\end{array}$ \\
\hline $15: 45$ & Neuropathie & Prof. Dan Ziegler, Düsseldorf \\
\hline $17: 15$ & Schwangerschaft & Dr. Helmut Kleinwechter, Kiel \\
\hline 18:15 & Fuß + pAVK & Prof. Maximilian Spraul, Rheine \\
\hline \multicolumn{3}{|c|}{ Samstag, 3. März 2012} \\
\hline 08:30 & Herz-Kreislauf & PD Dr. Michael Lehrke, Aachen \\
\hline 09:30 & Hypertonie & Prof. Roland Schmieder, Erlangen \\
\hline $11: 00$ & Psychosomatik & Prof. Ulrich T. Egle, Gengenbach \\
\hline $11: 45$ & Typ 2 Diabetes & Prof. Michael Stumvoll, Leipzig \\
\hline $14: 00$ & Nephropathie & Prof. Gunter Wolf, Jena \\
\hline $15: 00$ & ZNS & Prof. Frank Erbguth, Nürnberg \\
\hline
\end{tabular}

Die Veranstaltung wird von der LÄK Rheinland-Pfalz mit 16 CME-Fortbildungspunkten zertifiziert.

Ausführliche Informationen und Online-Anmeldung finden Sie unter www.diabetes-update.com.

\section{Wissenschaftliche Leitung}

Prof. Dr. Werner Scherbaum, Düsseldorf

Prof. Dr. Stephan Martin,

Düsseldorf

Prof. Dr. Michael Stumvoll, Leipzig

\section{Seminarort}

Rheingoldhalle, Rheinstraße 66 55116 Mainz

\section{Anmeldung/Teilnehmer- registrierung}

wikonect $\mathrm{GmbH}$

Tel.: 0611-204809-14

Fax: 0611-204809-10

www.diabetes-update.com

\section{Teilnahmegebühr}

$350,-€ \quad$ Reduziert bis 24.02.2012

400,- $€ \quad$ Regulär ab 25.02.2012 25,- $€$ Preisreduktion für Assistenzärzte*, Diabetesberater ${ }^{\star}$ und DGIM-Mitglieder*

\section{Team-Ticket für Klinik und} Praxis

500,- $€$ Team-Ticket 1 (1 Arzt +1 Assistenz, z. B. Assistenzarzt ${ }^{*}$, Diabetesberater ${ }^{\star}$ )

650,- $€$ Team-Ticket 2 (1 Arzt +2 Assistenzen, z. B. Assistenzarzt ${ }^{\star}$, Diabetesberater ${ }^{\star}$ )

Ab 25.02.2012 erhöhen sich die Preise der Team-Tickets um $50,-€$.

(*Nachweis erforderlich)

\section{Die Teilnahmegebühr} beinhaltet

- Seminarteilnahme an beiden Tagen

- Handbuch DIABETOLOGIE 2012

- Zugang zum Diabetes eUpdate mit allen Vorträgen inkl. Originalton der Referenten

- Pausenverpflegung an beiden Tagen

- Get-together am Freitagabend

\section{Konzeptentwicklung} und Veranstalter

med update $\mathrm{GmbH}$, Wiesbaden www.med-update.com 